interaction between tuberculosis infection and HIV infection in both developing and industrialised countries.

Preventive therapy and the place of BCG vaccination are discussed and control strategies for high- and low-prevalence areas are covered. Directly observed therapy is now being rediscovered as an effective means of treating patients where compliance is a problem either due to lifestyle (homelessness, drug addiction, alcoholism, etc.) or geography (rural areas in developing countries). The success achieved with this strategy in both developing and industrialised countries is one of the great hopes for the future.

Many chapters contain summaries in the form of 'Learning points' which are very helpful. Some of the chest radiographs do not reproduce well and there are a few typographical errors which have escaped the editorial red pen! However, this book would make an excellent addition to any bookshelf, and I would happily use it as my main reference source for tuberculosis.

H. MORRISON

\section{Mycoplasma protocols: Methods in Molecular Biology, volume 104}

Edited by Roger MiLes and RoBIN NiCHOLAS. 1998. ISBN 0-89603-525-5. Humana Press, New Jersey. Pp. 330. \$79.50.

Although part of a series covering methods in molecular biology, about half of this 33-chapter volume is actually directed at the laboratory isolation, propagation and identification of mycoplasmas of medical and veterinary interest. Media formulations and the treatment of specimens are explained simply and in great detail. This will be useful to laboratories carrying out the routine examination of submitted material or to laboratories with a limited knowledge of mycoplasmology, planning to introduce a diagnostic service. Of particular interest is the chapter on quality control testing, a subject often overlooked in many laboratory manuals.

Following the established form of the series, each chapter consists of a brief introduction to the technique, followed by a list of materials including the names of manufacturers, a methods section and, finally, of particular value, notes in which the authors give tips and point out pitfalls based on their practical experience.

To me, a number of chapters stand out as being especially interesting. Chapters 10 and 11 cover methods by which the metabolic activities of mycoplasmas may be studied. In the light of the recent publication of complete genome sequences for Mycoplasma genitalium and M. pneumoniae, the relevance of comparative genomic analysis to metabolic function is explained, together with some interesting examples. As more genomes are sequenced and become available, this new field is certain to expand.

The middle section of the book from chapters 12 to 16 provides detailed methodologies for some of the more specialised immunodiagnostic techniques which are not often performed but which can provide useful confirmatory tests in laboratories specialising in mycoplasmas. These include antigen detection with immune sera and monoclonal antibodies as well as in-situ immunohistochemical staining.

Chapters 17-22 deal with the characterisation of mycoplasmas by nucleic acid techniques. Methods based on the PCR include 16S rDNA sequencing, RAPD and RFLP fingerprinting. The latter two protocols are specifically aimed at the $M$. mycoides 'cluster' and may require some adjustment for use with other species. Although the preparation steps and precautions described are in most circumstances applicable to all PCRs, there could have been a more general approach, particularly with regard to alternative methods for the preparation of clinical samples, avoidance of contamination and removal of inhibitors. Chapters on DNA-DNA hybridisation and insertion sequence analysis are well covered but, surprisingly, pulsed-field gel electrophoresis either for strain charcterisation or genome size determination is not included.

Some of the more specialised DNA research techniques are given a detailed treatment in chapters 25-29. These methods are currently used on a regular basis in only a few specialist research departments. Perhaps these chapters will encourage more laboratories to attempt them.

Transformation in mycoplasmas is rarely achieved and in species where it is recognised, only low transformation frequencies are obtained and these may be very dependent on the method used. Chapter 25 gives four different methods. Similarly, transposon mutagenesis has rarely been successful with mycoplasmas and chapter 26 is particularly focused on the most successful system so far - the transfer of Tn916 from donor strains of Enterococcus faecalis to several species of mycoplasmas.

The detection of extrachromasomal elements in mycoplasmas is covered in chapter 27 , and although this is another rare event, it may be necessary to rule out the presence of plasmid or bacteriophage in certain circumstances.

One of the major problems facing the study of mycoplasmas is the inability to express gene products in the usual vector systems. This is largely due to the atypical codon usage of mycoplasmas. Chapters 28 and 29 describe some of the more recent methods for expressing mycoplasmal genes which have achieved some success.

The final three chapters deal with aspects of protein characterisation with particular emphasis on adhesins and surface antigens, including general methods for electrophoresis, Western blotting, screening expression libraries, monoclonal antibody production, labelling techniques and electron microscopy.

The value of this book lies in the amount of technical detail provided. Ideally, this should enable the interested investigator to carry out the protocols described without recourse to other publications. Although this may be an optimistic viewpoint, I feel that at least the reader will be encouraged to have a go. 